\title{
Adaptive Coordinated Control Strategy of Multi Manipulator System based on Multi-Agent
}

\author{
Nianen Zhu' ${ }^{1}$ Jiang $\operatorname{Han}^{2}$, Lian Xia ${ }^{2}$, Hui Liu ${ }^{1}$ \\ ${ }^{1}$ School of Mechatronics and Mould Engineering, Taizhou Vocational College of Science and \\ Technology, Taizhou, 318020, China \\ ${ }^{2}$ College of Mechanical Engineering, Hefei University of Technology, HeFei, 230009, China
}

Received: March 2, 2021. Revised: July 31, 2021. Accepted: August 26, 2021. Published: August 27, 2021.

\begin{abstract}
With people's increasing awareness of life and the increasing complexity of exploration in unknown environment, a single robot can not meet the increasing demand, including the price, flexibility and efficiency of robots. As a common mechanical control system in industrial production instead of human production, multi manipulator system can be applied in complex environment, multi task and other conditions. In order to settle the coordinated control fault of multi manipulator system, we study adaptive coordinated control strategy with the help of multi-agent research method in this paper, which can simplify the complexity of the problem and design an efficient and feasible system control protocol. The complex items in the multi manipulator system are treated as non affine systems. Using the design idea of non affine algorithm, combined with implicit function theorem and median theorem, the non affine system is transformed into affine systems, the controller is separated, and a distributed adaptive control strategy is designed. The results indicate that manipulator systems can effectively track the active manipulator system in finite time and the significance of the algorithm is proven by MATLAB simulation analysis.
\end{abstract}

Index Terms-Multi Manipulator System, Multi-Agent, PID control, Variable Structure Control, Position Tracking

\section{INTRODUCTION}

I recent years, there has not been clear and uniform definition for "the manipulator of the robot" in the world. Generally, the manipulator of the robot is an automation device which can simulate part of the action of human's hand and arm, capture, transport or manipulate tools according to predetermined program, track and other requirements[1]. But a "manipulator" generally has fixed hand, fixed action program or simple and variable program and an automation device generally used to fix station. Because the three kinds of automated or semi-automated devices of "the manipulator of the robot", "manipulator" and "operating machine" at home and abroad have certain similarities technically, sometimes it is difficult to clearly distinguish them. The manipulator is a dynamic system with complex multiple input and multiple output, highly nonlinear, time-varying and strong coupling. Its problem of control has been an active area of research of robotics[2-3].

As a common mechanical control system in industrial production instead of human production, multi manipulator system can be applied in complex environment, multi task and other conditions[4-6]. From the individual of the system, a single manipulator has certain local perception ability, autonomous movement ability and the ability to respond to the environment. In this way, the multi manipulator system has the same structural characteristics as the multi-agent system. Here, we use the multi-agent research method to simplify the complexity of the problem and design an efficient and feasible system control protocol.

Multi-agent is a new and cutting-edge complex interdisciplinary discipline. Multi-agent discipline has developed from simple linear, first-order and containing many constraints to nonlinear, high-order and other research systems [7-9]. Compared with a single agent, multi-agent has the advantages of stability and cooperation. At the same time, it also has the way of distributed control. It can solve the potential influencing factors such as packet loss, attenuation and communication failure in many bad situations, and has strong redundancy. In fact, in production and life, the controlled objects we get are nonlinear or have more complex forms on the basis of nonlinearity, so the nonlinear problem is an unavoidable problem. In addition, the communication between agents may also be nonlinear. When studying nonlinear multi-agent, we need to grasp the structure of each agent (i.e. topological information structure), dynamic equation and the function of the whole system. Therefore, it is very valuable to discuss the Adaptive Coordinated Control of distributed multi-agent nonlinear systems. 


\section{THE MATHEMATICAL MODEL OF THE CONTROLLED OBJEC}

The system of master slave robotic manipulator investigated in this paper is linked by two manipulators with the same structures through electrical signal. master robotic manipulator is directly controlled by the operator[10-11]. But slave robotic manipulator directly faces the object to be manipulated. Slave robotic manipulator gets the information such as position, posture from master slave robotic manipulator. At the same time, slave robotic manipulator sends master slave robotic manipulator part of the environmental information of the scene such as touch, taste and strength so that the operator has immersive feeling, thus ensuring the successful completion of operation.

The master/slave robot with 2-degree of freedom, two-link and rotary joints are studied in this paper, and the robot model is shown Figure 1.

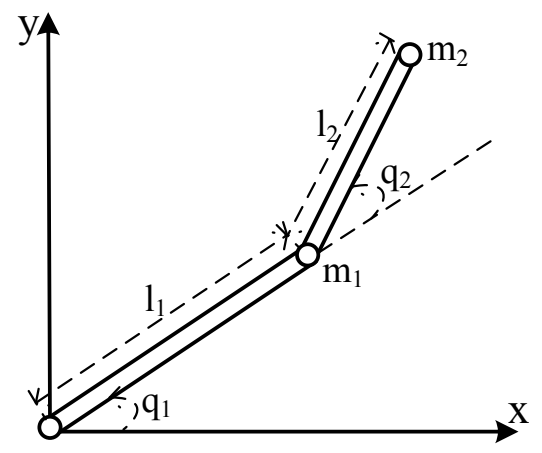

Fig. 1 The robot model with 2-degree of freedom, two-link and rotary joints

In Figure $1, l_{1}, l_{2}$ are the lengths of the two links, $m_{1}, m_{2}$ are the links' mass, $q_{1}, q_{2}$ are the joint angular positions of the two links.

The control of master slave robotic manipulator belongs to position - force hybrid control. There are two basic control requirements for it, one is the position of slave robotic manipulator $x_{\mathrm{s}}$ tracks the position of master robotic manipulator $x_{\mathrm{m}}$, the other is the grip of slave robotic manipulator $f_{\mathrm{s}}$ tracks the grip of master robotic manipulator $f_{\mathrm{m}}$, so that the operator can operate various operations of slave robotic manipulator on the scene from a place far away from the scene by operating slave robotic manipulator. The dynamic equation of master slave robotic manipulator can be expressed as

$$
\begin{gathered}
M_{\mathrm{m}} \frac{\mathrm{d}^{2} x_{\mathrm{m}}}{\mathrm{d} t^{2}}+b_{\mathrm{m}} \frac{\mathrm{d} x_{\mathrm{m}}}{\mathrm{d} t}=\tau_{\mathrm{m}}+f_{\mathrm{m}}+d_{\mathrm{m}} \\
M_{\mathrm{s}} \frac{\mathrm{d}^{2} x_{\mathrm{s}}}{\mathrm{d} t^{2}}+b_{\mathrm{s}} \frac{\mathrm{d} x_{\mathrm{s}}}{\mathrm{d} t}=\tau_{\mathrm{s}}-f_{\mathrm{s}}+d_{\mathrm{s}}
\end{gathered}
$$

where $M_{\mathrm{m}}$ and $M_{\mathrm{s}}$ are respectively the moment of inertia of master and slave robotic manipulator, $b_{\mathrm{m}}$ and $b_{\mathrm{s}}$ are respectively viscous friction coefficient of master and slave robotic manipulator, $f_{\mathrm{m}}$ is the grip that is applied to master robotic manipulator by operator, $f_{\mathrm{s}}$ is the reaction of grip that the objects to be operated applies to slave robotic manipulator, $\tau_{\mathrm{m}}$ and $\tau_{\mathrm{s}}$ are respectively the control moment of master robotic manipulator and slave robotic manipulator, $d_{\mathrm{m}}$ and $d_{\mathrm{s}}$ are respectively modeling uncertainties and external disturbances of master robotic manipulator and slave robotic manipulator. $\left|d_{\mathrm{s}}\right| \leq D, D$ is the set threshold.

The reaction of Slave Robotic Manipulator $f_{\mathrm{s}}$ can be expressed as

$$
f_{\mathrm{s}}=b_{\mathrm{w}} \frac{\mathrm{d} x_{\mathrm{s}}}{\mathrm{d} t}+c_{\mathrm{w}} x_{\mathrm{s}}
$$

where $b_{\mathrm{w}}$ is equivalent damping factor, $c_{\mathrm{w}}$ is equivalent elasticity coefficient.

Replace Equation (3) into Equation (2), the following can be given

$$
M_{\mathrm{s}} \frac{\mathrm{d}^{2} x_{\mathrm{s}}}{\mathrm{d} t^{2}}+\left(b_{\mathrm{s}}+b_{\mathrm{w}}\right) \frac{\mathrm{d} x_{\mathrm{s}}}{\mathrm{d} t}+c_{\mathrm{w}} x_{\mathrm{s}}=\tau_{\mathrm{s}}+d_{\mathrm{s}}
$$

\section{CONTROL LAW}

\section{A.Design of PID Control of Master Robotic Manipulator}

PID control is with flexible structural change, strong applicability, and excellent static performance[12]. It is insensitive to the change of the control object feature, and even if the mathematical model of the controlled object is imprecise, good control effect can also be obtained. For most of the control objects, basically satisfactory results can be achieved by using PID control.

In the control system of master slave robotic manipulator, the dynamic equation of Master Robotic Manipulator is Equation (1). PID control strategy is used to control it. Assuming tracking error of strength can be expressed as

$$
e_{\mathrm{f}}=f_{\mathrm{m}}-f_{\mathrm{s}}
$$

Taking PID control law as

$$
\tau_{\mathrm{m}}=k_{\mathrm{p}} e_{\mathrm{f}}+k_{\mathrm{i}} \int e_{\mathrm{f}} \mathrm{d} t+k_{\mathrm{d}} \dot{e}_{\mathrm{f}}
$$

If there is a theoretical method to determine the PID parameters in the PID parameter setting, of course, this condition is the most ideal. But, in practical applications, the trial and error method is more often used to determine the parameters of PID. Increasing the proportion factor $\mathrm{P}$ will 
generally accelerate the system's response[13]. And if there is a static error, the static error tends to reduce. But the system takes a longer time to eliminate static error. Increasing the derivative time $\mathrm{D}$ will make the overshoot reduce and the stability increase for the system. But system's capability of suppressing disturbance weakens. When the trial and error method is used, the above parameters can be utilized to refer to the impacts and trends of system's control process, and the adjustment of the parameter can perform proportion first, integrate after performing proportion and then perform differential.

\section{B.Design of Variable Structure Control of Slave Robotic Manipulator}

Variable Structure Control (VSC) is a class of nonlinear control [14-16]. Its principle is as follows. A phase trajectory is artificially set crosses the equilibrium point. Through appropriate design, the state point of the system gradually stablizes in the equilibrium point along this phase trajectory. Sliding Mode Control is the most mainstream design method in VSC, so VSC can be generally named as sliding mode control (SMC). Sliding mode can be designed, and it has nothing to do with the parameters of the object and disturbance, it responses rapidly, it is insensitive to variations of the parameter and disturbances (robustness), it does not need online recognition and it is simple to be realized in physics.

Considering the control law of manipulator, this paper uses the method of sliding mode control. Three elements are controlled by sliding mode variable structure: The conditions of accessibility is met, the sliding mode is existential, and the mode of sliding mode has asymptotic stability and good dynamic quality.

Taking tracking error of location as

$$
e=x_{\mathrm{m}}-x_{\mathrm{s}}
$$

then

$$
\dot{e}=\dot{x}_{\mathrm{m}}-\dot{x}_{\mathrm{s}}
$$

Design sliding function as

$$
s=c e+\dot{e}, c>0
$$

Because

$$
\begin{aligned}
\ddot{e} & =\ddot{x}_{\mathrm{m}}-\ddot{x}_{\mathrm{s}} \\
& =\ddot{x}_{\mathrm{m}}-\left(t_{\mathrm{s}}+d_{\mathrm{s}}-\left(b_{\mathrm{s}}+b_{\mathrm{w}}\right) \dot{x}_{\mathrm{s}}-c_{\mathrm{w}} x_{\mathrm{s}}\right) / M_{\mathrm{s}}
\end{aligned}
$$

then

$$
\begin{aligned}
\ddot{s} & =c \dot{e}+\ddot{e} \\
& =c \dot{e}+\ddot{x}_{\mathrm{m}}-\left(\tau_{\mathrm{s}}+d_{\mathrm{s}}-\left(b_{\mathrm{s}}+b_{\mathrm{w}}\right) \dot{x}_{\mathrm{s}}-c_{\mathrm{w}} x_{\mathrm{s}}\right) / M_{\mathrm{s}}
\end{aligned}
$$

Sliding mode control law is designed as

$$
\begin{gathered}
\tau_{\mathrm{s}}=M_{\mathrm{s}}\left(c \dot{e}+\ddot{x}_{\mathrm{m}}\right)+\left(b_{\mathrm{s}}+b_{\mathrm{w}}\right) \dot{x}_{\mathrm{s}}+c_{\mathrm{w}} x_{\mathrm{s}}+ \\
\left(D_{\mathrm{s}}+\eta\right) \operatorname{sgn} s \\
\dot{s}=-\frac{\left(D_{\mathrm{s}}+\eta\right) \operatorname{sgn} s+d_{\mathrm{s}}}{M_{\mathrm{s}}}
\end{gathered}
$$

Constructing Lyapunov function

$$
V=\frac{1}{2} s^{2}
$$

Then

$$
s \dot{s}=-\frac{\left(D_{\mathrm{s}}+\eta\right)|s|+d_{\mathrm{s}} s}{M_{\mathrm{s}}} \leq-\frac{\eta|s|}{M_{\mathrm{s}}}
$$

That is, $\dot{V}<0$, when the condition is achieved, the state point will approach the switching surface.

\section{C.Simulation}

The dynamic equations of master slave robotic manipulator are Equation (1) and Equation (2), taking $M_{\mathrm{m}}=10.768, \quad b_{\mathrm{m}}=420.7 \quad, \quad M_{\mathrm{s}}=0.768 \quad, \quad b_{\mathrm{s}}=417.5$, $b_{\mathrm{w}}=30, c_{\mathrm{w}}=1500, d_{\mathrm{m}}=0.2 \cos (t), d_{\mathrm{s}}=0.2 \sin (t)$.

Taking command of the strength $f_{\mathrm{m}}$ as square wave signal, control law can be expressed as Equation (6), control parameters can be taken as $k_{\mathrm{p}}=30, k_{\mathrm{i}}=3, k_{\mathrm{d}}=0.5$. In the control law of slidino mode Fouation(12) $D=1.0 \quad n=0.10 \quad r=50$

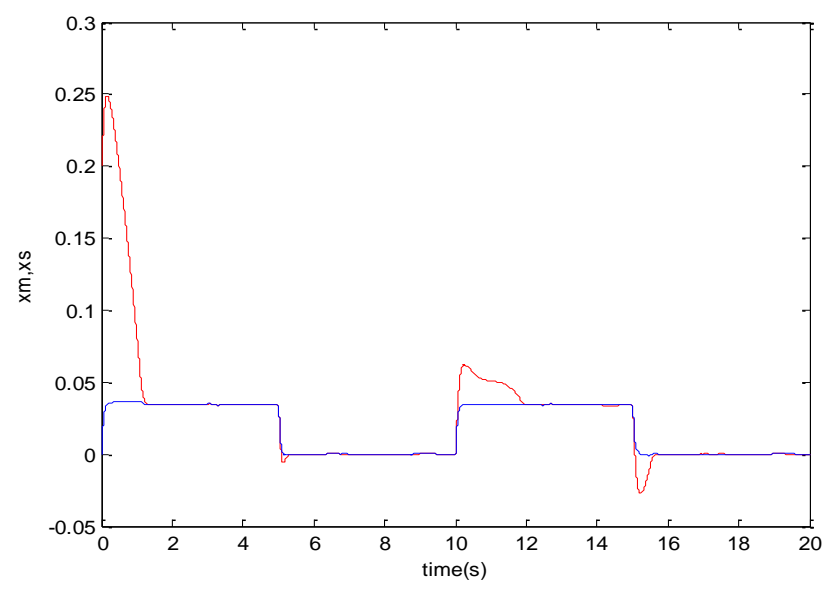

Fig 2. Location tracking curve of Slave Robotic Manipulator 


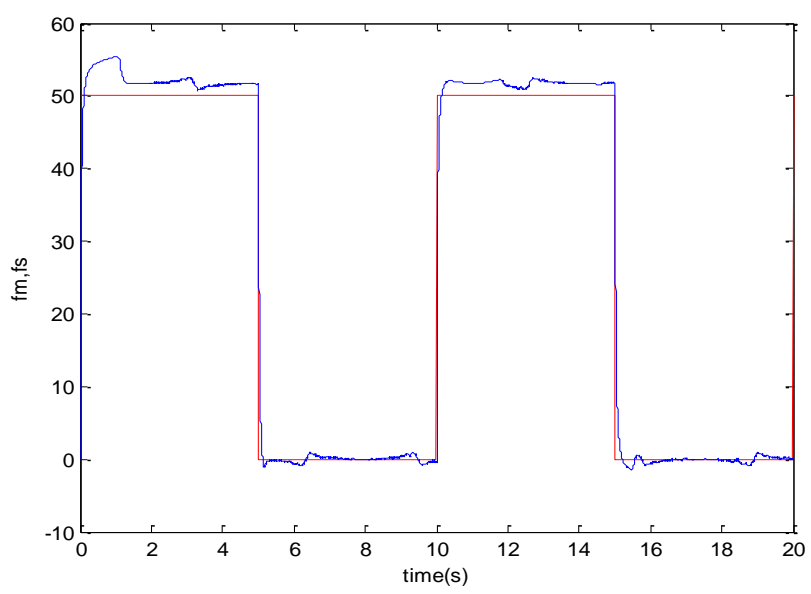

Fig 3. Strength tracking curve of Slave Robotic Manipulator

It can be seen from Figure 1 and Figure 2 that position and force signals are able to track the variations of Master Robotic Manipulator. But the overshoot in Figure 1 is larger and there is subtle fluctuation in Figure 2.

For the tracking control of Master Slave Robotic Manipulator, a controller strategy is designed. For Master Robotic Manipulator, for a classical control method of PID is mainly used, for Slave Robotic Manipulator, control method of sliding mode is mainly used. And the Lyapunov function is designed to validate stability of the designed controller.

\section{Distributed AdAPTIVE COORDINATED CONTROL OF MULTI MANIPULATOR SYSTEM}

\section{A.Problem Description}

$n$ driven manipulator systems are considered, and the system model is as follows

$$
D \ddot{q}+C \dot{q}+G=\tau
$$

Defining the active manipulator system can be expressed as

$$
q_{d}=x_{0}
$$

At the same time, the position status is defined as

$$
\dot{q}_{i}=x_{i}^{1}
$$

and the speed status is defined as

$$
\dot{x}_{i}=x_{i}^{2}
$$

then

$$
\begin{gathered}
\dot{x}_{i}^{2}=\ddot{q}_{i} \\
\ddot{q}_{i}=M_{i}^{-1}\left(\tau-C_{i} \dot{q}-G_{i}\right)=\dot{x}_{i}^{2}
\end{gathered}
$$

\section{B. Design of Distributed Adaptive Coordination Controller}

An adaptive controller is designed for the follower agents in the agent system to solve the synchronization problem between them and the leader.

For the synchronization problem of multi manipulator system, the distributed adaptive coordination controller is designed as follows

$$
\tau_{i}=-K r_{i}-f_{i}\left(x_{i}\right)+\frac{\lambda}{d_{i}+b_{i}} e_{i}^{2}
$$

where $f_{i}\left(x_{i}\right)$ is the approximation function of the nonlinear part in the $i$ th manipulator system, $K$ is the feedback gain, and $e_{i}^{2}$ is the speed error of the $i$ th manipulator system.

\section{Simulation}

In order to prove the correctness of the theory, a kind of two joint manipulator system is selected as the simulation analysis object, and the goal is to realize their adaptive coordinated tracking control. We select the tracking signal of the active manipulator.

$\left\{\begin{array}{l}q_{d 1}=1+0.2 * \cos \left(p i^{*} t\right) \\ q_{d 2}=1+0.2 * \cos (0.5 * p i * t)\end{array}\right.$

For the driven manipulator system, we select the following parameters

$M_{i}\left(q_{i}\right)=\left(\begin{array}{cc}d_{1}+d_{2}+2 d_{3} \cos \left(\theta_{i 2}\right) & d_{1}+d_{2}+2 d_{3} \cos \left(\theta_{i 2}\right) \\ d_{2}+d_{3} \cos \left(\theta_{i 2}\right) & d_{2}\end{array}\right)$

$C_{i}\left(q_{i}, \dot{q}_{i}\right)=\left(\begin{array}{cc}-d_{3} \dot{\theta}_{i 2} \sin \left(\theta_{i 2}\right) & -d_{3}\left(\dot{\theta}_{i 1}+\dot{\theta}_{i 2}\right) \sin \left(\theta_{i 2}\right) \\ -d_{3} \dot{\theta}_{i 1} \sin \left(\theta_{i 2}\right) & 0\end{array}\right)$

Select $a_{1}=10, a_{2}=5, K=50$ and $p_{i}>0$, and the output simulation results of each adaptive controller are as follows.

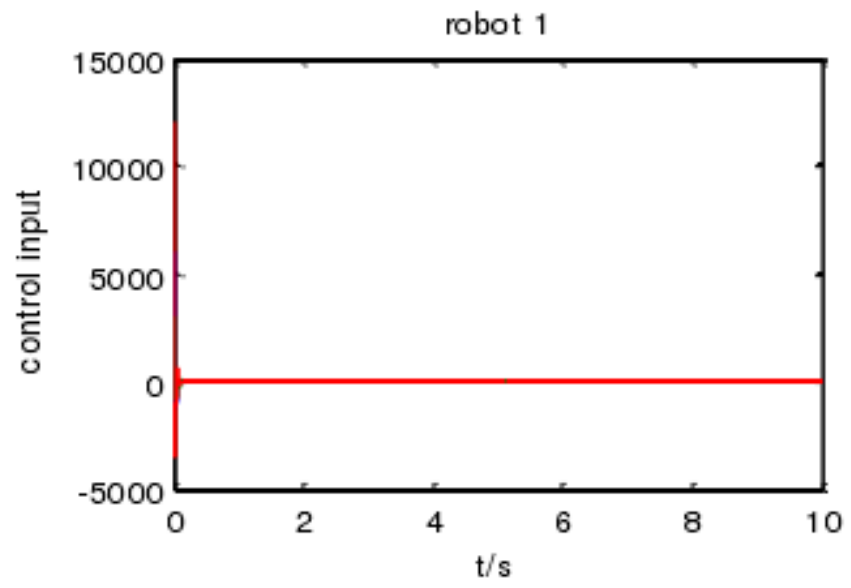


robot 2

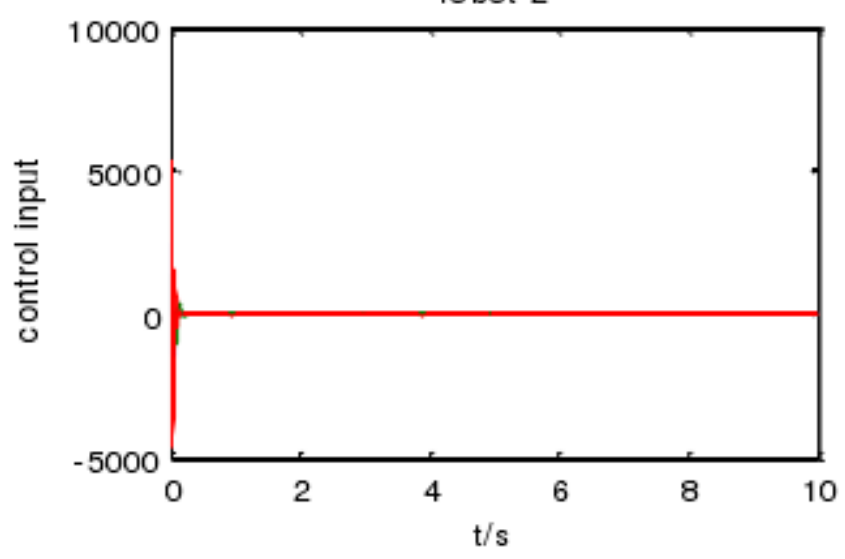

robot 3

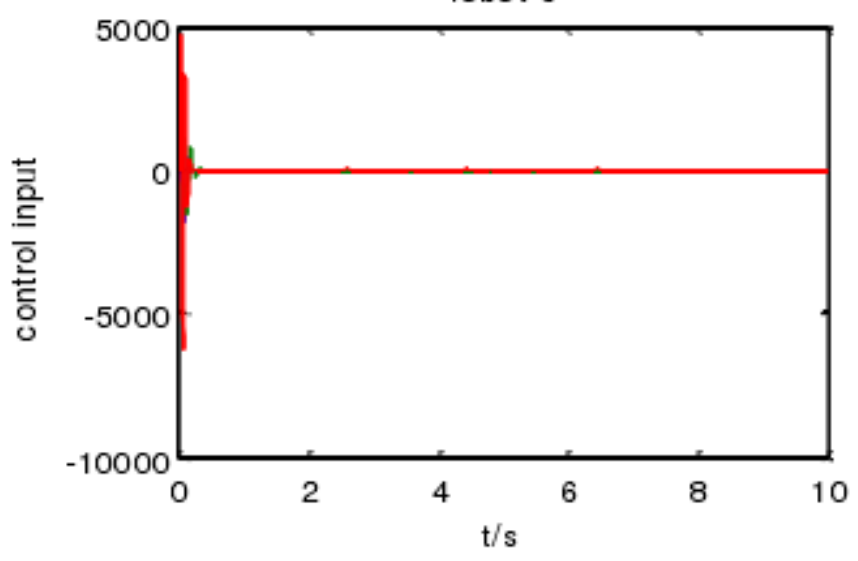

robot 4

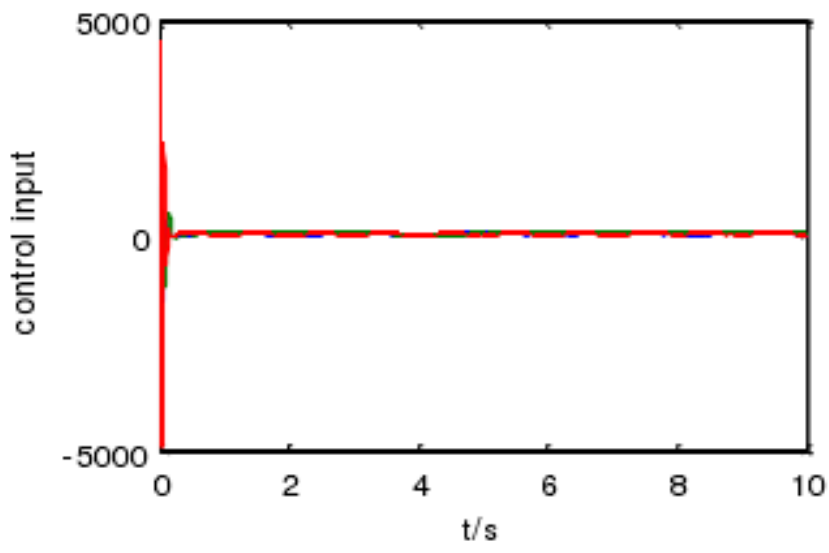

Fig. 4 Control input of the system

The position tracking analysis results of the four manipulator systems are as follows.

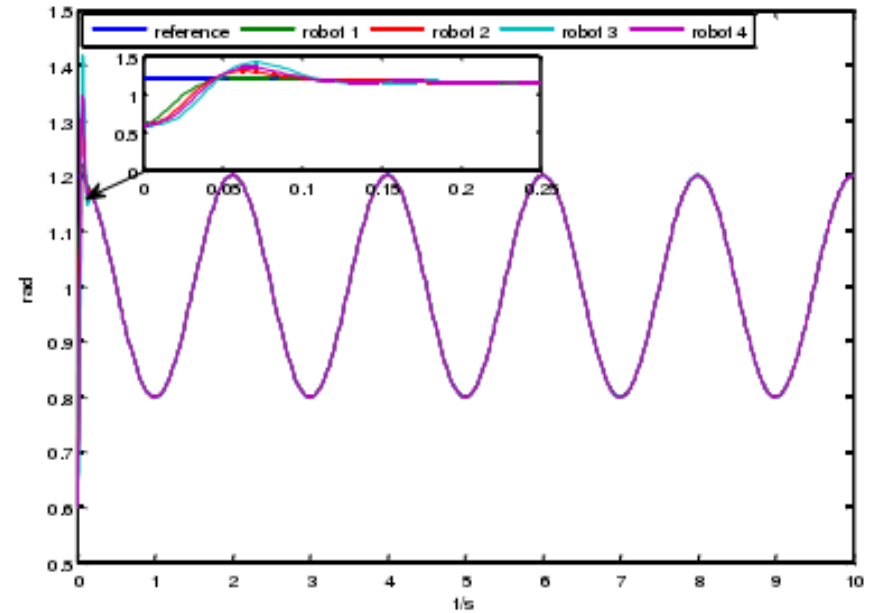

Fig. 5 The position tracking for pitch 1 of the 4 mechanical arm system

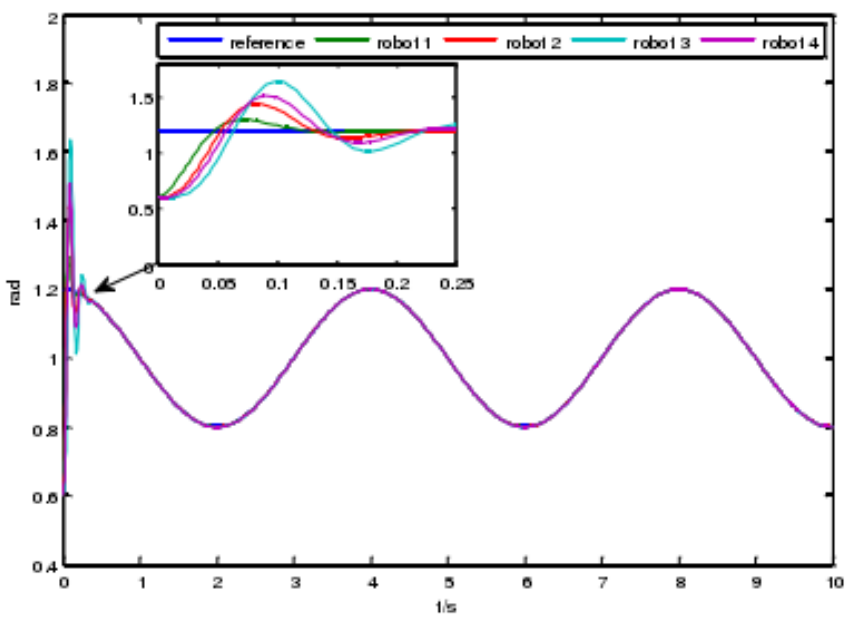

Fig. 6 The position tracking for pitch 2 of the 4 mechanical arm system

Although this kind of system is not a non affine system, we can adopt the conventional method to deal with nonlinear non affine problems, which is the median theorem, and then design a distributed adaptive controller to make the whole system track our given reference signal. Finally, five two joint robots are selected, which can only use local information to design distributed controller. Through Matlab example simulation, the feasibility of the algorithm is analyzed to verify the position error and velocity error.

\section{CONCLUSION}

This paper applies proposed control algorithm to multi manipulator systems based on the distributed Adaptive Coordinated Control. Firstly, the tracking problem of high-order model nonlinear multi-agent system is studied based on non affine multi-agent system. For high-order affine model, implicit function theorem and median theorem are introduced. At the same time, based on the approximation ability of neural network, a distributed adaptive coordination controller is designed. For multi manipulator system, we extend the idea of non affine multi-agent to it. Then, simulation analysis is finished for two kinds of systems. 
Due to the structural similarity between multi manipulator system and multi-agent system, multi-agent system has become a hot spot in modern control field. Rich research results have been achieved in both theoretical research and practical application. Their research ranges from simple linear systems to complex nonlinear systems, from basic first order to deep second order, and even higher order; Among them, there are relatively few research results on non affine systems in nonlinear systems, which still has great potential and is worth studying. For the multi manipulator system, the dynamic multi-agent non affine system in the directed communication topology is considered first in theory, and the distributed adaptive controller is designed, but all of them are carried out under assumptions and constraints. On the other hand, we select the verification of the algorithm under an ideal condition, which is based on certain assumptions. In view of the shortcomings in this paper and combined with several contents that the author thinks have research value, we give the following summary.

Firstly, the theory of a class of multi-agent non affine systems is studied. In this paper, the knowledge of non affine systems in some ideal environments is studied, and some results are obtained. However, this algorithm does not have certain redundancy. For example, when the system fails or the detection situation is not ideal, the whole system will have some unpredictable results. Therefore, for this phenomenon, we can add fault-tolerant control to the control algorithm. In addition, in the processing of high-order nonlinear functions, scholars at home and abroad have many advanced algorithms for these systems, such as backstepping, fuzzy adaptive control algorithms and so on. The introduction of these algorithms is also a challenging and valuable topic.

Secondly, in the application of the algorithm, we introduce an ideal multi manipulator system, and this system is not a non affine system, but regards the complex nonlinear part as a non affine system. A more advanced algorithm can be chosen to optimize the stability of the system. These are also some research topics with great potential and practical significance.

Finally, the actual platform is constructed. The distributed adaptive coordinated tracking control algorithm for a class of non affine systems proposed in this paper is in the form of software simulation, but there is no practical application platform to verify. In practice, there are many unstable conditions that will limit the effect of our algorithm. Therefore, we should pay more attention to the research on the construction of the actual platform, and many practical examples will have different unknown effects, so that we can improve the algorithm. This aspect is also of great practical value.

\section{ACKNOWLEDGMENT}

This work was supported by Teacher Professional Development Project of 2019 Domestic visiting scholar of universities by Zhejiang Provincial Department of Education (Collaborative manufacturing based on $5 \mathrm{G}$ and multi-agent technology, FX2019117).

\section{REFERENCES}

[1] Ni F, Wo S , Li Z . Coordinated control strategy of UPQC by dynamic collaborative task-solving scheme based on multi-agent system. Energy Systems, 2021:1-19.

[2] Li C , Zhang S, Li J , et al. Coordinated control strategy of multiple energy storage power stations supporting black-start based on dynamic allocation. The Journal of Energy Storage, 2020, 31:101683.

[3] Zhang Z, Qian J , Fang C , et al. Coordinated Control of Distributed Traffic Signal Based on Multiagent Cooperative Game. Wireless Communications and Mobile Computing, 2021, 2021:1-13.

[4] Wu Y, Cheng Y, Wang Y . Research on a Multi-Motor Coordinated Control Strategy Based on Fuzzy Ring Network Control. IEEE Access, 2020, PP(99): 1-1.

[5] Qing A I, Liu T, Y Yin, et al. Frequency CoordinatedControl Strategy of HVDCSending System with Wind Power Based on Situation Awareness. IET Generation Transmission \& Distribution, 2020, 14(16).

[6] H Kim, Lee J, Lee J, et al. Novel Coordinated Control Strategy of BESS and PMSG-WTG for Fast Frequency Response. 2021. vol. 23, no. 5, pp. 744-754.

[7] Liang C , Xu X, Wang F, et al. Coordinated control strategy for mode transition of DM-PHEV based on MLD[J]. Nonlinear Dynamics, 2021, 103(5).

[8] Wu T, Li Q, Bao X B, et al. Time-delay signature concealment in chaotic secure communication system combining optical intensity with phase feedback. Optics Communications, 2020, 475:126042.

[9] Wen S , Guo G . Sampled-Data Control for Connected Vehicles With Markovian Switching Topologies and Communication Delay. IEEE Transactions on Intelligent Transportation Systems, 2020, 21, pp. 2930-2942.

[10] Chung H, Ma Q, Sayginer M, et al. A Packaged 0.01-26-GHz Single-Chip SiGe Reflectometer for Two-Port Vector Network Analyzers. IEEE Transactions on Microwave Theory and Techniques, 2020, $\mathrm{PP}(99): 1-15$.

[11]Lucjan Setlak, Rafal Kowalik, Dynamics of the Designed Robotic Manipulator in the CAD Program, WSEAS Transactions on Applied and Theoretical Mechanics, Volume 14, 2019, Art. \#7, pp. 66-74.

[12] Khaled Mustafa, Abdulgani Albagoul, Mustafa Saad, Design of Fuzzy Adaptive PID Controller for Nonlinear Multivariable Process, WSEAS Transactions on Systems, Volume 18, 2019, Art. \#33, pp. 262-269.

[13] GK Mertin, M Oldenburger, E Richter, Revised theory of entropy and reversible energy flow in galvanic cells. Journal of Power Sources, 2021, 482:228813.

[14] Van Den Berg J, Abbeel P, Goldberg K. LQG-MP: Optimized path planning for robots with motion uncertainty and imperfect state information. The International Journal of Robotics Research, 2019, 30(7): 895-913.

[15] Badmos T A, Omolaye P O, Mebawondu J, et al. Robot Path Planning Performance Evaluation of a Dynamic Environment. IOSR Journal of Electronics and Communication Engineering, 2020, 13, pp.19-26.

[16] Atia M G B, El-Hussieny H, Salah O. A Supervisory-Based Collaborative Obstacle-Guided Path Refinement Algorithm for Path Planning in Wide Terrains. IEEE Access, 2020,170: 257-266.

\section{Creative Commons Attribution License 4.0 (Attribution 4.0 International, CC BY 4.0)}

This article is published under the terms of the Creative Commons Attribution License 4.0

https://creativecommons.org/licenses/by/4.0/deed.en_US 